\title{
Invariant Manifold Based Reduced-order Observer Design for Nonlinear Systems
}

\author{
Dimitrios Karagiannis, Daniele Carnevale, Alessandro Astolfi
}

\begin{abstract}
The problem of constructing globally convergent, reduced-order observers for general nonlinear systems is addressed. It is shown that an asymptotic estimate of the unknown states can be obtained by rendering attractive an appropriately selected (invariant) manifold in the extended state space. Current results on nonlinear observer design require that the nonlinearities appearing in the system equations are either linear functions of the unmeasured states or monotonic functions of a linear combination of the states. In this paper we relax these two assumptions by allowing for a wider class of nonlinearities to appear in the system equations. The proposed approach is applied on several examples including a perspective vision system and a general two-degrees-of-freedom mechanical system.
\end{abstract}

Index Terms-observers, mechanical systems, nonlinear systems

\section{INTRODUCTION}

$\mathbf{T}$ HE problem of constructing observers for nonlinear systems has received a lot of attention due to its importance in practical applications, where some of the states may not be available for measurement. In the case of linear systems, a complete theory on asymptotic (reduced-order) observers can be found in [1], while an observer with finite-time convergence has been recently developed in [2].

The classical approach to nonlinear observer design consists in finding a transformation that linearises the plant up to an output injection term and then applying standard linear observer design techniques. The existence of such a transformation, however, relies on a set of stringent assumptions [3], [4] which are hard to satisfy in practice. These have been recently relaxed in [5]. Lyapunov-like conditions for the existence of a nonlinear observer with asymptotically stable error dynamics have been given in [6]. An observer for uniformly observable nonlinear systems in canonical form has been developed in [7], [8] based on a global Lipschitz condition and a gain assignment technique. Some extensions to this result, which avoid the transformation to canonical form and allow for more flexibility in the selection of the observer gain, have been proposed in [9]. More recently, in [10] conditions for the existence of a linear observer with a nonlinear output mapping have been given in terms of the

D. Karagiannis is with the Department of Electrical and Electronic Engineering, Imperial College London, SW7 2AZ, United Kingdom. (E-mail: d.karagiannis@imperial.ac.uk).

D. Carnevale is with the Dipartimento di Informatica, Sistemi e Produzione, Università di Roma "Tor Vergata", 00133 Roma, Italy. (E-mail: carnevale@disp.uniroma2.it).

A. Astolfi is with the Department of Electrical and Electronic Engineering, Imperial College London, SW7 2AZ, United Kingdom, and with the Dipartimento di Informatica, Sistemi e Produzione, Università di Roma "Tor Vergata”, 00133 Roma, Italy. (E-mail: a.astolfi@imperial.ac.uk). local solution of a partial differential equation (PDE), thus extending Luenberger's early ideas [1] to the nonlinear case. Extensions to this fundamental result have been developed in [11], [12], see also [13]. A globally convergent reduced-order observer for systems in canonical form has been proposed in [14] using the notion of output-to-state stability, whereas a high-gain design for a similar class of systems that presumes a bound on the system trajectories has been proposed in [15], see [16] for a survey on high-gain observers.

A design which can deal with a general class of nonlinear systems without resorting to high gain has been proposed in [17], see also [18], [19], under the restrictions that the nonlinearities appearing in the system equations are monotonic functions of a linear combination of the states and that the linear part of the observer error system can be rendered strictly positive real (SPR) by solving a linear matrix inequality (LMI).

In the present paper we relax the above assumptions by following the general approach introduced in [20], where the observer design problem is formulated as a problem of rendering attractive an appropriately selected invariant manifold in the extended state-space of the plant and the observer. ${ }^{1}$ In particular, we allow for non-monotonic nonlinearities to appear in the unmeasured state dynamics with coefficients that are not necessarily constant but may depend on the system output. Interestingly, even in the special case where these coefficients are constant and the nonlinearities are monotonic, the result is more general than the one in [17] (see the example of Section III).

The proposed extension is mainly achieved by exploiting the (monotonic) nonlinearities appearing in the output dynamics in order to stabilise the observer error system. From a passivity point of view, this approach ensures that the "shortage" of passivity due to the non-monotonic terms is compensated for by an "excess" of passivity due to output injection in the observer error dynamics. Additionally, we show that for systems with finite escape time the output injection term can be in some cases shaped so that the observer converges in finite time (namely at the time of the escape).

The method is used to obtain new solutions to two practical problems. The first is the problem of estimating the three-dimensional motion of an object using two-dimensional images obtained from a single camera. The second is the problem of estimating the velocities of a general two-degreesof-freedom (2DOF) mechanical system by measuring the

\footnotetext{
${ }^{1}$ This approach, which is inspired by the adaptive stabilisation tools in [21], was first introduced for systems that are linear in the unmeasured states in [22].
} 
positions.

The paper is organised as follows. In Section II we formulate the observer design problem and propose a general methodology for constructing asymptotically convergent (reduced-order) observers. This is illustrated with a practical example. In Section III a particular class of systems with non-monotonic nonlinearities, which allows us to obtain more constructive results, is discussed and an academic example for which the method of [17] is not applicable is provided. An application to 2DOF mechanical systems is considered in Section IV. Finally, Section V concludes the paper with some summarising remarks. (Preliminary versions of the general results presented here can be found in the conference papers [20], [23].)

\section{MAIN RESUlT}

We consider nonlinear, time-varying systems described by equations of the form

$$
\begin{aligned}
& \dot{y}=f_{1}(y, x, t) \\
& \dot{x}=f_{2}(y, x, t)
\end{aligned}
$$

where $y \in \mathbb{R}^{m}$ is the measured part of the state and $x \in \mathbb{R}^{n}$ is the unmeasured part of the state. It is assumed that the vector fields $f_{1}(\cdot)$ and $f_{2}(\cdot)$ are forward complete, i.e. trajectories starting at time $t_{0}$ are defined for all times $t \geq t_{0}$. (We will show later that this assumption can be removed under certain conditions, see Corollary 2.)

Definition 1: The dynamical system

$$
\dot{\xi}=\alpha(y, \xi, t)
$$

with $\xi \in \mathbb{R}^{p}, p \geq n$, is called an observer for the system (1)(2), if there exist mappings

$$
\beta(\cdot): \mathbb{R}^{m} \times \mathbb{R}^{p} \times \mathbb{R} \rightarrow \mathbb{R}^{p} \quad \text { and } \quad \phi_{y, t}(\cdot): \mathbb{R}^{n} \rightarrow \mathbb{R}^{p}
$$

with $\phi_{y, t}(\cdot)$ parameterised by $y$ and $t$ and left-invertible ${ }^{2}$ such that the manifold

$\mathcal{M}=\left\{(y, x, \xi, t) \in \mathbb{R}^{m} \times \mathbb{R}^{n} \times \mathbb{R}^{p} \times \mathbb{R}: \beta(y, \xi, t)=\phi_{y, t}(x)\right\}$

has the following properties.

(i) All trajectories of the extended system (1)-(3) that start on the manifold $\mathcal{M}$ remain there for all future times, i.e. $\mathcal{M}$ is positively invariant.

(ii) All trajectories of the extended system (1)-(3) that start in a neighbourhood of $\mathcal{M}$ asymptotically converge to $\mathcal{M}$.

The above definition is in the spirit of the definition given in [6] and implies that an asymptotic estimate of the state $x$ is given by $\phi_{y, t}^{L}(\beta(y, \xi, t))$, where $\phi_{y, t}^{L}$ denotes a left-inverse of $\phi_{y, t}$.

\footnotetext{
${ }^{2} \mathrm{~A}$ mapping $\phi_{y, t}(\cdot): \mathbb{R}^{n} \rightarrow \mathbb{R}^{p}$ (parameterised by $y$ and $t$ ) is left-invertible if there exists a mapping $\phi_{y, t}^{L}(\cdot): \mathbb{R}^{p} \rightarrow \mathbb{R}^{n}$ such that $\phi_{y, t}^{L}\left(\phi_{y, t}(x)\right)=x$, for all $x \in \mathbb{R}^{n}$ (and for all $y$ and $t$ ). Recall that a mapping is left invertible if and only if it is injective.
}

Remark 1: One could alternatively, and equivalently, define the manifold $\mathcal{M}$ as

$\mathcal{M}=\left\{(y, x, \xi, t) \in \mathbb{R}^{m} \times \mathbb{R}^{n} \times \mathbb{R}^{p} \times \mathbb{R}: x=\phi_{y, t}^{L}(\beta(y, \xi, t))\right\}$.

The difference between the parameterisations in (4) and in (5) is in the definition of the estimation error, and hence of the error dynamics. In the former case, the natural selection for the estimation error is

$$
z=\beta(y, \xi, t)-\phi_{y, t}(x)
$$

whereas in the latter case it is

$$
\tilde{z}=\phi_{y, t}^{L}(\beta(y, \xi, t))-x .
$$

Existence of an injection gain rendering the zero equilibrium of the $z$ dynamics globally (uniformly) asymptotically stable does not imply existence of an injection gain achieving the same objective for the zero equilibrium of the $\tilde{z}$ dynamics. This issue is illustrated in Example 1 and Remark 4.

We now present the main result of the paper, namely a general tool for constructing a nonlinear observer of the form given in Definition 1.

Proposition 1: Consider the system (1)-(3) and suppose that there exist mappings $\beta(\cdot): \mathbb{R}^{m} \times \mathbb{R}^{p} \times \mathbb{R} \rightarrow \mathbb{R}^{p}$ and $\phi_{y, t}(\cdot): \mathbb{R}^{n} \rightarrow \mathbb{R}^{p}$, with a left-inverse $\phi_{y, t}^{L}(\cdot): \mathbb{R}^{p} \rightarrow \mathbb{R}^{n}$, such that the following hold.

(A1) For all $y, \xi$ and $t$,

$$
\operatorname{det}\left(\frac{\partial \beta}{\partial \xi}\right) \neq 0
$$

(A2) The system

$$
\begin{aligned}
\dot{z}= & -\frac{\partial \beta}{\partial y}\left(f_{1}(y, \hat{x}, t)-f_{1}(y, x, t)\right) \\
& +\left.\frac{\partial \phi_{y, t}}{\partial y}\right|_{x=\hat{x}} f_{1}(y, \hat{x}, t)-\frac{\partial \phi_{y, t}}{\partial y} f_{1}(y, x, t) \\
& +\left.\frac{\partial \phi_{y, t}}{\partial x}\right|_{x=\hat{x}} f_{2}(y, \hat{x}, t)-\frac{\partial \phi_{y, t}}{\partial x} f_{2}(y, x, t) \\
& +\left.\frac{\partial \phi_{y, t}}{\partial t}\right|_{x=\hat{x}}-\frac{\partial \phi_{y, t}}{\partial t}
\end{aligned}
$$

with $\hat{x}=\phi_{y, t}^{L}\left(\phi_{y, t}(x)+z\right)$, has an asymptotically stable equilibrium at $z=0$, uniformly in $x, y$ and $t$.

Then the system (3) with

$$
\begin{aligned}
\alpha(y, \xi, t)= & -\left(\frac{\partial \beta}{\partial \xi}\right)^{-1}\left(\frac{\partial \beta}{\partial y} f_{1}(y, \hat{x}, t)+\frac{\partial \beta}{\partial t}\right. \\
& -\left.\frac{\partial \phi_{y, t}}{\partial y}\right|_{x=\hat{x}} f_{1}(y, \hat{x}, t) \\
& \left.-\left.\frac{\partial \phi_{y, t}}{\partial x}\right|_{x=\hat{x}} f_{2}(y, \hat{x}, t)-\left.\frac{\partial \phi_{y, t}}{\partial t}\right|_{x=\hat{x}}\right)
\end{aligned}
$$

where $\hat{x}=\phi_{y, t}^{L}(\beta(y, \xi, t))$, is a (reduced-order) observer for the system (1)-(2).

Proof: Consider the variable

$$
z=\beta(y, \xi, t)-\phi_{y, t}(x)
$$


where $\beta(\cdot)$ is a continuously differentiable function such that (A1) holds. Note that the Euclidean norm $|z|$ represents the distance at time $t$ of the system trajectories from the manifold $\mathcal{M}$ defined in (4). The dynamics of $z$ are given by

$$
\begin{aligned}
\dot{z}= & \frac{\partial \beta}{\partial y} f_{1}(y, x, t)+\frac{\partial \beta}{\partial \xi} \alpha(y, \xi, t)+\frac{\partial \beta}{\partial t}-\frac{\partial \phi_{y, t}}{\partial y} f_{1}(y, x, t) \\
& -\frac{\partial \phi_{y, t}}{\partial x} f_{2}(y, x, t)-\frac{\partial \phi_{y, t}}{\partial t} .
\end{aligned}
$$

Substituting the function $\alpha(\cdot)$ from (7) and noting that by assumption (A1) this function is well-defined, yields the dynamics (6). It follows from (A2) that the distance $|z|$ from the manifold $\mathcal{M}$ converges asymptotically to zero. Note, moreover, that $\mathcal{M}$ is invariant, i.e. if $z(t)=0$ for some $t$, then $z(\tau)=0$ for all $\tau>t$. Hence, by Definition 1, the system (3) with $\alpha(\cdot)$ given by (7) is a (reduced-order) observer for (1)(2).

Remark 2: Proposition 1 provides an implicit description of the observer dynamics (3) in terms of the mappings $\beta(\cdot), \phi_{y, t}(\cdot)$ and $\phi_{y, t}^{L}(\cdot)$ which must then be selected to satisfy (A2). ${ }^{3}$ Hence the problem of constructing an observer for the system (1)-(2) is reduced to the problem of rendering the zero equilibrium of system (6) asymptotically stable by assigning the functions $\beta(\cdot), \phi_{y, t}(\cdot)$ and $\phi_{y, t}^{L}(\cdot)$. This nonstandard stabilisation problem can be extremely difficult to solve, since, in general, it relies on the solution of a set of partial differential equations (or inequalities). However, in many cases of practical interest, these equations are solvable, as demonstrated in the following example.

Remark 3: The proposed observer design method relies on the construction of a manifold with specific properties, namely invariance and attractivity. Invariant and attractive manifolds in observer design have been introduced in the work of Luenberger [1] and have been recently exploited, for nonlinear systems, in [10], [11], [12] and [14].

In [10] an observer is defined (on the basis of Luenberger's ideas) as a linear asymptotically stable system, driven by the available measurements and with a nonlinear output map, and the state estimate is obtained by inversion of this output map. Under non-resonance conditions, and exploiting Lyapunov's auxiliary theorem, it is proved that the interconnection of the system, the state of which has to be estimated, with the observer has a locally well-defined invariant and attractive manifold, with the property that the estimation error is zero on the manifold. The non-resonance conditions of [10] have been relaxed in [11], and a global version of these results has been given in [12] under certain observability and completeness assumptions. In all these works the observer has linear dynamics, the (local or global) existence and invariance of the manifold is guaranteed by non-resonance conditions or completeness assumptions, and the attractivity is implied by stability of the observer dynamics.

In [14] the observer dynamics are linear modulo a dynamic scaling which depends upon an estimate of the norm of the state, the manifold and the output map are described by linear

\footnotetext{
${ }^{3}$ Note, however, that the function $\alpha(\cdot)$ in (7) renders the manifold $\mathcal{M}$ invariant for any mappings $\beta(\cdot)$ and $\phi_{y, t}(\cdot)$.
}

maps with coefficients depending on the dynamic scaling (note that the linear structure is a consequence of the use of the observability canonical form), and global attractivity is proved by means of Lyapunov arguments.

In contrast, in the proposed approach, a parameterised description of the manifold is given (hence there is no existence issue), and the observer dynamics are selected to render the manifold invariant. The crucial issue is therefore the attractivity of the manifold, which has to be achieved by a proper selection of the functions $\beta(\cdot), \phi_{y, t}(\cdot)$ and $\phi_{y, t}^{L}(\cdot)$, as discussed in Remark 2.

Example 1 (Range estimation): We consider the problem of estimating the range of an object moving in the threedimensional space by observing the motion of its projected feature on the two-dimensional image space of a camera. Previous solutions to this problem, which typically arises in machine vision as well as target tracking, can be found in [24], [25], [26], [27].

Assuming that the motion of the object is described by linear (time-varying) dynamics and taking a perspective model for the camera yields the system (see [27] for details)

$$
\begin{aligned}
& \dot{y}=h_{1}(y, t)+g(y, t) x \\
& \dot{x}=h_{2}(y, t) x-b_{3}(t) x^{2}
\end{aligned}
$$

where $y=\left[y_{1}, y_{2}\right]^{\top} \in \mathbb{R}^{2}$ are the measurable co-ordinates on the image space, $x \in \mathbb{R}$ is the inverse range of the object, and

$$
\begin{aligned}
h_{1}(y, t)= & {\left[\begin{array}{cc}
a_{11}(t)-a_{33}(t) & a_{12}(t) \\
a_{21}(t) & a_{22}(t)-a_{33}(t)
\end{array}\right] y+\left[\begin{array}{l}
a_{13}(t) \\
a_{23}(t)
\end{array}\right] } \\
& -y y^{\top}\left[\begin{array}{l}
a_{31}(t) \\
a_{32}(t)
\end{array}\right] \\
h_{2}(y, t)= & -\left(a_{31}(t) y_{1}+a_{32}(t) y_{2}+a_{33}(t)\right) \\
g(y, t)= & {\left[\begin{array}{l}
b_{1}(t)-b_{3}(t) y_{1} \\
b_{2}(t)-b_{3}(t) y_{2}
\end{array}\right] . }
\end{aligned}
$$

It is assumed that $a_{i j}(t), b_{i}(t)$ and $y(t)$ are bounded, $a_{i j}(t)$ and $b_{i}(t)$ are differentiable, and the "instantaneous observability" condition

$$
|g(y, t)| \geq \epsilon>0
$$

holds, for some $\epsilon$ and for all $t$ and $y$.

For simplicity let $\phi_{y, t}(x)=\varepsilon(y, t) x$, where $\varepsilon(\cdot) \neq 0$ is a function to be determined, and consider an observer of the form given in Proposition 1, namely

$$
\begin{aligned}
\dot{\xi}= & -\left(\frac{\partial \beta}{\partial \xi}\right)^{-1}\left(\frac{\partial \beta}{\partial y}\left(h_{1}(y, t)+g(y, t) \hat{x}\right)+\frac{\partial \beta}{\partial t}\right. \\
& -\frac{\partial \varepsilon}{\partial y}\left(h_{1}(y, t)+g(y, t) \hat{x}\right) \hat{x}-\frac{\partial \varepsilon}{\partial t} \hat{x} \\
& \left.-\varepsilon(y, t)\left(h_{2}(y, t) \hat{x}-b_{3}(t) \hat{x}^{2}\right)\right) \\
\hat{x}= & \frac{1}{\varepsilon(y, t)} \beta(y, \xi, t) .
\end{aligned}
$$

From (6) the dynamics of the error

$$
z=\beta(y, \xi, t)-\varepsilon(y, t) x=\varepsilon(y, t)(\hat{x}-x)
$$


are given by

$$
\begin{aligned}
\dot{z}= & -\frac{\partial \beta}{\partial y} g(y, t)(\hat{x}-x) \\
& +\left(\frac{\partial \varepsilon}{\partial y} h_{1}(y, t)+\varepsilon(y, t) h_{2}(y, t)\right)(\hat{x}-x) \\
& +\left(\frac{\partial \varepsilon}{\partial y} g(y, t)-\varepsilon(y, t) b_{3}(t)\right)\left(\hat{x}^{2}-x^{2}\right) \\
= & -\left(\frac{\partial \beta}{\partial y} g(y, t)-\frac{\partial \varepsilon}{\partial y} h_{1}(y, t)-\varepsilon(y, t) h_{2}(y, t)\right) \frac{1}{\varepsilon(y, t)} z \\
& +\left(\frac{\partial \varepsilon}{\partial y} g(y, t)-\varepsilon(y, t) b_{3}(t)\right)\left(\hat{x}^{2}-x^{2}\right) .
\end{aligned}
$$

The observer design problem is now reduced to finding functions $\beta(\cdot)$ and $\varepsilon(\cdot) \neq 0$ that satisfy assumptions (A1)-(A2) of Proposition 1 . In view of (14) this can be achieved by solving the PDEs

$$
\begin{aligned}
\frac{\partial \beta}{\partial y} g(y, t)-\frac{\partial \varepsilon}{\partial y} h_{1}(y, t) & \\
-\varepsilon(y, t) h_{2}(y, t) & =\kappa(y, t) \varepsilon(y, t) \\
\frac{\partial \varepsilon}{\partial y} g(y, t)-\varepsilon(y, t) b_{3}(t) & =0
\end{aligned}
$$

for some $\kappa(\cdot)$. From (16) we obtain the solution

$$
\varepsilon(y, t)=-\frac{1}{|g(y, t)|}
$$

which by (11) is well-defined and non-zero for all $y$ and $t$. Let

$$
\kappa(y, t)=\lambda|g(y, t)|^{3}-\frac{\partial \varepsilon}{\partial y} h_{1}(y, t) \varepsilon(y, t)^{-1}-h_{2}(y, t)
$$

and note that, by (11) and the fact that $a_{i j}(t), b_{i}(t)$ and $y$ are bounded, we can always find $\lambda>0$ (sufficiently large) such that $\kappa(\cdot)>\underline{\kappa}$, for some $\underline{\kappa}>0$. The PDE (15) is now reduced to

$$
\frac{\partial \beta}{\partial y} g(y, t)=-\lambda|g(y, t)|^{2}
$$

which can be solved for $\beta(\cdot)$ yielding

$$
\begin{aligned}
\beta(y, \xi, t)= & \frac{\lambda}{2}\left(\left(y_{1}^{2}+y_{2}^{2}\right) b_{3}(t)-2 b_{1}(t) y_{1}-2 b_{2}(t) y_{2}\right) \\
& +c(\xi, t)
\end{aligned}
$$

where $c(\cdot)$ is a free function. Selecting $c(\xi, t)=\xi$ ensures that assumption (A1) is satisfied. Substituting the above expressions into (14) yields the system

$$
\dot{z}=-\kappa(y, t) z
$$

which has a uniformly asymptotically stable equilibrium at zero, hence assumption (A2) holds.

Remark 4: It is worth comparing the foregoing result with the one in [27], where a semi-global observer for the perspective system (9)-(10) was obtained using a similar procedure but with $\phi_{y, t}(x)$ the identity mapping (i.e. with $\varepsilon(y, t)=1$ ). In the present design, on the other hand, we are able to obtain a global observer by assigning the function $\varepsilon(y, t)$, hence the mapping $\phi_{y, t}(x)$, to satisfy the PDE (16).

\section{FURTHER RESULTS}

The generality of Proposition 1 comes at a price: it does not provide a constructive way of finding a function $\beta(\cdot)$ that satisfies (A2). In this section we attempt to give a better insight into the design of this function by studying a more specific class of systems. In particular, we show that the monotonic nonlinearities appearing in the output dynamics can be exploited to stabilise the zero equilibrium of the observer error system even in the presence of non-monotonic nonlinearities, thus extending the result in [17].

We consider a class of nonlinear systems described by equations of the form

$$
\begin{aligned}
& \dot{y}=F_{1}(y) \gamma(C y+H x)+g_{1}(y, u) \\
& \dot{x}=F_{2}(y) \gamma(C y+H x)+\delta(y, x)+g_{2}(y, u)
\end{aligned}
$$

where $y \in \mathbb{R}^{m}$ is the measurable output, $x \in \mathbb{R}^{n}$ is the unmeasured state, $u \in \mathbb{R}^{r}$ is the input, and $\gamma(\cdot) \in \mathbb{R}^{p}$ is a vector with each element a nonlinear function of a linear combination of the states, i.e.

$$
\gamma_{i}=\gamma_{i}\left(C_{i} y+H_{i} x\right)
$$

where $C_{i}$ and $H_{i}$ denote the rows of $C$ and $H$, respectively. We assume that the functions $\gamma(\cdot)$ and $\delta(\cdot)$ are continuously differentiable and that each $\gamma_{i}$ is non-decreasing, i.e.

$$
(a-b)\left(\gamma_{i}(a)-\gamma_{i}(b)\right) \geq 0
$$

for all $a, b \in \mathbb{R}$. Note that, comparing with the class of systems considered in [17], there is an additional nonlinearity $\delta(\cdot)$ in (18) which is not necessarily monotonic and the matrices $F_{1}$ and $F_{2}$ generally depend on the output $y$.

For simplicity we fix the mapping $\phi_{y, t}(\cdot)$ in Definition 1 to be the identity and choose $p=n$, hence the problem considered is to find a reduced-order observer of the form

$$
\dot{\xi}=\alpha(y, \xi) \quad \hat{x}=\beta(y, \xi)
$$

with $\xi \in \mathbb{R}^{n}$.

Remark 5: It should be mentioned that the result of [17] relies strongly on the matrices $F_{1}$ and $F_{2}$ being constant. Moreover, the system dynamics must include a linear (detectable) part which plays an essential role in the stabilisation of the observer error dynamics. As we will see, our construction does not require a linear part, since stabilisation of the observer error dynamics will be achieved by exploiting the monotonic nonlinearities contained in the vector $\gamma(\cdot)$ - which may also include linear terms.

Note that, when $\delta(y, x) \equiv 0$, the system (17)-(18) can be rewritten in the form

$$
\begin{aligned}
& \dot{y}=A_{1}(y) x+G_{1}(y) \bar{\gamma}(\bar{C} y+\bar{H} x)+g_{1}(y, u) \\
& \dot{x}=A_{2}(y) x+G_{2}(y) \bar{\gamma}(\bar{C} y+\bar{H} x)+g_{2}(y, u)
\end{aligned}
$$

where $G_{1}(y)=\left[F_{1}(y),-A_{1}(y)\right], G_{2}(y)=\left[F_{2}(y),-A_{2}(y)\right]$ and

$$
\bar{\gamma}(\bar{C} y+\bar{H} x)=\left[\begin{array}{c}
\gamma(C y+H x) \\
x
\end{array}\right] .
$$

If, in addition, $F_{1}, F_{2}, A_{1}$ and $A_{2}$ are constant, then (21)-(22) coincides with the systems considered in [17]. 
Example 2: To motivate our approach (and highlight the differences from the one in [17]), we consider a simple example described by the two-dimensional system

$$
\begin{aligned}
& \dot{y}=x+x^{3}-y \\
& \dot{x}=x^{2}+u
\end{aligned}
$$

and the problem of constructing a (reduced-order) observer for the unmeasured state $x$. In an attempt to apply the methodology in [17], we first rewrite the system in the form

$$
\begin{aligned}
& \dot{y}=A_{1} x+G_{1} \gamma(x)-y \\
& \dot{x}=A_{2} x+G_{2} \gamma(x)+u
\end{aligned}
$$

where $\gamma(x)=\left[x^{3}, x+x^{2}+x^{3}\right]^{\top}$ satisfies the monotonicity condition (19), and $A_{1}=1, G_{1}=[1,0], A_{2}=-1$, and $G_{2}=[-1,1]$. Defining the error $z=\xi+B y-x$ and the observer dynamics

$\dot{\xi}=\left(A_{2}-B A_{1}\right)(\xi+B y)+\left(G_{2}-B G_{1}\right) \gamma(\xi+B y)+u+B y$

yields the error system

$$
\dot{z}=\left(A_{2}-B A_{1}\right) z+\left(G_{2}-B G_{1}\right)(\gamma(x+z)-\gamma(x))
$$

which is asymptotically stable if there exist constants $\nu_{1}>0$, $\nu_{2}>0$ and $B$ such that the following conditions hold ${ }^{4}$

$$
A_{2}-B A_{1} \leq 0 \quad G_{2}-B G_{1}=\left[-\nu_{1},-\nu_{2}\right] .
$$

It can be readily seen that the last condition cannot be satisfied, since it requires $\nu_{2}=-1$.

The method in [17] failed in this example because it was not possible to assign the "output injection gain" $B$ to render the error system passive with respect to each one of the elements of the vector $\gamma(x+z)-\gamma(x)$, which are passive by assumption (19). Obviously, this passivity requirement becomes more restrictive as the dimension of the vector $\gamma(\cdot)$ increases. However, since passivity is not a necessary condition for stability, there may still exist a $B$ such that the error system is asymptotically stable.

We now show that such a solution can be constructed by means of Proposition 1 . Towards this end, let $\beta(y, \xi)=$ $\xi+f(y)$, which satisfies condition (A1), and consider the observer (7) which in this case becomes

$$
\begin{aligned}
\alpha(y, \xi)= & (\xi+f(y))^{2}-\frac{\partial f}{\partial y}\left(\xi+f(y)+(\xi+f(y))^{3}\right) \\
& +u+\frac{\partial f}{\partial y} y
\end{aligned}
$$

Substituting into the dynamics of the error $z=\hat{x}-x=$ $\xi+f(y)-x$ yields the system (6), which can be written as

$$
\begin{aligned}
\dot{z} & =\left((x+z)^{2}-x^{2}\right)-\frac{\partial f}{\partial y}\left(z+(x+z)^{3}-x^{3}\right) \\
& =\left(2 x+z-\frac{\partial f}{\partial y}\left(1+3 x^{2}+3 x z+z^{2}\right)\right) z \\
& \triangleq \lambda(y, x, z) z .
\end{aligned}
$$

The aim now is to find $f(y)$ such that $\lambda(y, x, z)<0$ for all $y, x$ and $z$. Rewriting $\lambda(\cdot)$ as a quadratic polynomial in $z$ and its discriminant as a polynomial in $x$ we obtain the conditions

$$
\frac{\partial f}{\partial y}>0 \text { and } 1-3\left(\frac{\partial f}{\partial y}\right)^{2}<0
$$

Hence, selecting $f(y)=B y$, with $B>1 / \sqrt{3}$, ensures that the error system (25) is uniformly globally asymptotically stable, hence Proposition 1 holds.

Remark 6: Notice that for $u(t)=0$ and $x(0)>0$ the states of the system (23)-(24) escape to infinity in finite time $t_{e}=1 / x(0)$. Nevertheless, it is possible to prove finite-time convergence of the observer error. To this end, define a scaled time variable $\tau$ such that $\mathrm{d} \tau / \mathrm{d} t=1+x^{2}$ which implies

$$
\tau=\int_{0}^{t}\left(1+x(\zeta)^{2}\right) \mathrm{d} \zeta
$$

and note that $\tau \rightarrow \infty$ as $t \rightarrow t_{e}$. From (25), the dynamics of the observer error with respect to the new time variable $\tau$ can be written as

$$
\frac{\mathrm{d} z}{\mathrm{~d} \tau}=\dot{z} \frac{\mathrm{d} t}{\mathrm{~d} \tau}=\frac{\lambda(y, x, z)}{1+x^{2}} z .
$$

Note that the term $\lambda(y, x, z) /\left(1+x^{2}\right)$ is strictly negative even when $x(\tau) \rightarrow \infty$, hence

$$
\lim _{\tau \rightarrow \infty} z(\tau)=\lim _{\tau \rightarrow \infty}(\hat{x}(\tau)-x(\tau))=0
$$

which, in turn, implies $\lim _{t \rightarrow t_{e}}(\hat{x}(t)-x(t))=0$, i.e. the manifold $\mathcal{M}$ in (4) is reached in finite time (namely at the time of escape $t_{e}$ ).

The following proposition provides a generalisation of the above design for the class of systems (17)-(18).

Proposition 2: Consider the system (17)-(18) with states $y(t)$ and $x(t)$ defined for all $t \geq 0$ and define two matrices $\Gamma(y, x, z)$ and $\Delta(y, x, z)$ such that

$$
\begin{aligned}
\Gamma(y, x, z) H z & =\gamma(C y+H(x+z))-\gamma(C y+H x) \\
\Delta(y, x, z) z & =\delta(y, x+z)-\delta(y, x) .
\end{aligned}
$$

Suppose that we can find a positive definite matrix $P$ and a function $\beta(y, \xi): \mathbb{R}^{m} \times \mathbb{R}^{n} \rightarrow \mathbb{R}^{n}$, with $\operatorname{det}(\partial \beta / \partial \xi) \neq 0$, that satisfy the matrix inequality

$$
\Lambda(y, x, z)^{\top} P+P \Lambda(y, x, z)<0
$$

where

$$
\Lambda(y, x, z)=\left(F_{2}(y)-\frac{\partial \beta}{\partial y} F_{1}(y)\right) \Gamma(y, x, z) H+\Delta(y, x, z)
$$

for all $y, x$ and $z$. Then there exists a reduced-order observer of the form (20) such that

$$
\lim _{t \rightarrow \infty}(\hat{x}(t)-x(t))=0 .
$$

Proof: Consider the system (20) and define the error variable $z=\hat{x}-x=\beta(y, \xi)-x$, whose dynamics are described by the equation

$$
\begin{aligned}
\dot{z}= & \frac{\partial \beta}{\partial \xi} \alpha(y, \xi)+\frac{\partial \beta}{\partial y}\left(F_{1}(y) \gamma(C y+H x)+g_{1}(y, u)\right) \\
& -F_{2}(y) \gamma(C y+H x)-\delta(y, x)-g_{2}(y, u) .
\end{aligned}
$$


Assigning the observer dynamics as in (7), namely

$$
\begin{aligned}
\alpha(y, \xi)= & \left(\frac{\partial \beta}{\partial \xi}\right)^{-1}\left(F_{2}(y) \gamma(C y+H \hat{x})+\delta(y, \hat{x})\right. \\
& \left.+g_{2}(y, u)-\frac{\partial \beta}{\partial y}\left(F_{1}(y) \gamma(C y+H \hat{x})+g_{1}(y, u)\right)\right)
\end{aligned}
$$

and noting that $\hat{x}=x+z$ yields the error system

$$
\begin{aligned}
\dot{z} & =\left(F_{2}(y)-\frac{\partial \beta}{\partial y} F_{1}(y)\right) \Gamma(y, x, z) H z+\Delta(y, x, z) z \\
& =\Lambda(y, x, z) z
\end{aligned}
$$

which, from (26), is uniformly globally asymptotically stable with the Lyapunov function $V(z)=z^{\top} P z$. As a result, Proposition 1 holds. Moreover, if $\beta(y, \xi)$ is invertible with respect to $\xi$, then the observer state $\xi(t)$ is bounded whenever $y(t)$ and $x(t)$ are bounded.

It is apparent from the construction given in the proof of Proposition 2 that the observer problem is reduced to the problem of finding a function $\beta(\cdot)$ that solves the (partial) differential inequality (26). In the special case of the class of systems considered in [17], with the additional restriction $\beta(y, \xi)=\xi+B y$, where $B$ is constant, this inequality reduces to the LMI given in [17], as the following corollary shows.

Corollary 1: Consider the system (21)-(22) with states $y(t)$ and $x(t)$ defined for all $t \geq 0$, where $\bar{\gamma}(\cdot)$ satisfies the condition (19). Suppose that we can find a positive definite matrix $P$, a function $\beta(y)$, a constant $\nu>0$ and a diagonal matrix $N>0$ that satisfy the matrix inequality

$$
\left[\begin{array}{c}
\left(A_{2}(y)-\frac{\partial \beta}{\partial y} A_{1}(y)\right)^{\top} P+P\left(A_{2}(y)-\frac{\partial \beta}{\partial y} A_{1}(y)\right)+\nu I \\
\left(G_{2}(y)-\frac{\partial \beta}{\partial y} G_{1}(y)\right)^{\top} P+N \bar{H} \\
\left.P\left(G_{2}(y)-\frac{\partial \beta}{\partial y} G_{1}(y)\right)+\bar{H}^{\top} N\right] \leq 0 .(27) \\
0
\end{array}\right.
$$

Then there exists a reduced-order observer of the form (20) such that

$$
\lim _{t \rightarrow \infty}(\hat{x}(t)-x(t))=0
$$

Proof: To begin with, note that the system (21)-(22) is a special case of the system (17)-(18) with $F_{1}(y)=$ $\left[G_{1}(y), A_{1}(y)\right], F_{2}(y)=\left[G_{2}(y), A_{2}(y)\right]$ and

$$
\gamma(C y+H x)=\left[\begin{array}{c}
\bar{\gamma}(\bar{C} y+\bar{H} x) \\
x
\end{array}\right] \text {. }
$$

Define a (diagonal) matrix $\bar{\Gamma}(y, x, z)$ such that

$$
\bar{\Gamma}(y, x, z) \bar{H} z=\bar{\gamma}(\bar{C} y+\bar{H}(x+z))-\bar{\gamma}(\bar{C} y+\bar{H} x)
$$

and note that

$$
\Gamma(y, x, z)=\left[\begin{array}{cc}
\bar{\Gamma}(y, x, z) & 0_{p \times n} \\
0_{n \times p} & I
\end{array}\right] \quad H=\left[\begin{array}{c}
\bar{H} \\
I
\end{array}\right] .
$$

The matrix inequality (26) can now be rewritten as

$$
(\star)^{\top} P+P(\star)<0
$$

where

$$
\star=\left(G_{2}(y)-\frac{\partial \beta}{\partial y} G_{1}(y)\right) \bar{\Gamma}(y, x, z) \bar{H}+A_{2}(y)-\frac{\partial \beta}{\partial y} A_{1}(y) .
$$

It remains to show that (27) implies (28), hence the result in Proposition 2 is applicable. This follows directly from noting that (27) implies

$$
\begin{gathered}
\left(A_{2}(y)-\frac{\partial \beta}{\partial y} A_{1}(y)\right)^{\top} P+P\left(A_{2}(y)-\frac{\partial \beta}{\partial y} A_{1}(y)\right)<0 \\
P\left(G_{2}(y)-\frac{\partial \beta}{\partial y} G_{1}(y)\right)=-\bar{H}^{\top} N
\end{gathered}
$$

and using the fact that, by condition (19), $z^{\top} \bar{\Gamma}(y, x, z) \bar{H} z \geq$ 0 , for all $y, x$ and $z$.

Remark 7: In the special case in which the matrices $A_{1}, A_{2}$, $G_{1}$ and $G_{2}$ are constant, (27) reduces to the LMI proposed in [17] by selecting $\beta(y)=B y$ with $B$ constant.

The result in Proposition 2 is based on the assumption that the system trajectories exist for all times. The following corollary shows that for a system with finite escape time (such as the one considered in Example 2) the observer can be made to converge in finite time.

Corollary 2: Consider the system (17)-(18) with states $y(t)$ and $x(t)$ maximally defined in $\left[0, t_{e}\right)$, for some $t_{e}>0$. Suppose that the assumptions of Proposition 2 hold and, moreover, there exists a function $\mu(x, y) \geq 1$ and a constant $\nu>0$ such that

$$
\frac{1}{\mu(x, y)}\left(\Lambda(y, x, z)^{\top} P+P \Lambda(y, x, z)\right) \leq-\nu I
$$

for some positive definite matrix $P$, and along any trajectory

$$
\lim _{t \rightarrow t_{e}} \int_{0}^{t} \mu(x(\zeta), y(\zeta)) \mathrm{d} \zeta=\infty .
$$

Then there exists a reduced-order observer of the form (20) such that

$$
\lim _{t \rightarrow t_{e}}(\hat{x}(t)-x(t))=0
$$

Proof: Define a scaled time variable $\tau$ such that

$$
\frac{\mathrm{d} \tau}{\mathrm{d} t}=\mu(x, y) \Longrightarrow \tau=\int_{0}^{t} \mu(x(\zeta), y(\zeta)) \mathrm{d} \zeta
$$

and note that, from (30), $\tau \rightarrow \infty$ as $t \rightarrow t_{e}$. Following the construction of the proof of Proposition 2, the observer error dynamics can be rewritten as

$$
\dot{z}=\frac{\mathrm{d} z}{\mathrm{~d} \tau} \frac{\mathrm{d} \tau}{\mathrm{d} t}=\Lambda(y, x, z) z
$$

which implies

$$
\frac{\mathrm{d} z}{\mathrm{~d} \tau}=\frac{1}{\mu(x, y)} \Lambda(y, x, z) z
$$

Note that, from (29), the above system is uniformly asymptotically stable with the Lyapunov function $V(z)=z^{\top} P z$, which implies that $\lim _{\tau \rightarrow \infty}(\hat{x}(\tau)-x(\tau))=0$, hence (31) holds. 


\section{APPLICATION TO 2DOF MECHANICAL SYSTEMS}

In this section we use the approach introduced above to construct globally asymptotically convergent observers for general Euler-Lagrange systems with two degrees of freedom, where the objective is to estimate the velocities by measuring the positions. This is a challenging problem since the dynamics involved are highly nonlinear with non-monotonic (quadratic) nonlinearities. An intrinsic local observer for this class of systems has been developed in [28] based on a Riemannian structure, while a global observer has been proposed in [29] albeit for a narrower class of systems that can be rendered linear in the unmeasured states via a transformation.

Following the proposed methodology, we first construct an output injection function $\beta(\cdot)$ by solving a set of partial differential equations and then ensure the convergence of the observer error by means of a quadratic Lyapunov function (see Proposition 2). As special cases we consider a two-link manipulator [30] and the well-known "ball and beam" system [31], [32]. The features of the proposed observers are shown via simulations.

\section{A. Model description}

Consider a 2DOF mechanical system whose dynamics are described by the Euler-Lagrange equations

$$
\frac{\mathrm{d}}{\mathrm{d} t} \frac{\partial \mathcal{L}}{\partial \dot{q}}(q, \dot{q})-\frac{\partial \mathcal{L}}{\partial q}(q, \dot{q})=\tau-F(q, \dot{q})
$$

where $q=\left[q_{1}, q_{2}\right]^{\top} \in \mathbb{R}^{2}$ is the vector of the (measurable) joint position variables, $\tau \in \mathbb{R}^{2}$ the vector of the motor torques and $F(q, \dot{q})$ represents the dissipative forces. The Lagrangian function $\mathcal{L}(q, \dot{q})=\mathcal{T}(q, \dot{q})-\mathcal{V}(q)$ is defined as the difference between the kinetic energy $\mathcal{T}(q, \dot{q})=\frac{1}{2} \dot{q}^{\top} M(q) \dot{q}$ and the potential energy $\mathcal{V}(q)$. We consider a tree-like articulated twobody system whose inertia matrix has the form

$$
M(q)=\left[\begin{array}{cc}
m_{11}\left(q_{2}\right) & m_{12}\left(q_{2}\right) \\
m_{12}\left(q_{2}\right) & m_{22}
\end{array}\right]
$$

with the standard assumptions $M(q)=M(q)^{\top}$ and $M(q)>$ 0 , for all $q$. The Euler-Lagrange equations (32) can also be written as

$$
M(q) \ddot{q}+C(q, \dot{q}) \dot{q}+G(q)=\tau-F(q, \dot{q})
$$

where the vector $G(q)$ accounts for the potential forces and the matrix $C(q, \dot{q})$ represents the Coriolis and centripetal forces and is derived using Christoffel symbols [33] as

$$
C(q, \dot{q})=\left[\begin{array}{cc}
\frac{1}{2} m_{11}^{\prime}\left(q_{2}\right) \dot{q}_{2} & \frac{1}{2} m_{11}{ }^{\prime}\left(q_{2}\right) \dot{q}_{1}+m_{12}{ }^{\prime}\left(q_{2}\right) \dot{q}_{2} \\
-\frac{1}{2} m_{11}{ }^{\prime}\left(q_{2}\right) \dot{q}_{1} & 0
\end{array}\right]
$$

where $m_{i, j}{ }^{\prime}$ denotes the first derivative of $m_{i, j}$ with respect to $q_{2}$. Note that the matrix $C(q, \dot{q})$ is linear in the second argument, i.e. the identity

$$
C(q, x+z)=C(q, x)+C(q, z)
$$

holds for all $q, x$ and $z$. Defining the states $y=q$ and $x=\dot{q}$, the system (34) can be rewritten in the form (1)-(2), namely

$$
\begin{aligned}
\dot{y} & =x \\
M(y) \dot{x} & =-C(y, x) x-F(y, x)-G(y)+\tau .
\end{aligned}
$$

\section{B. Observer design}

We now proceed to the design of a reduced-order observer of the form (3) for the velocity vector $x$ using the result in Section II. Let $\phi_{y, t}(x)=T(y) x$, where $T(y)$ is a $2 \times 2$ transformation matrix to be determined, and define the error variable

$$
z=\beta(y, \xi)-T(y) x=T(y)(\hat{x}-x)
$$

whose dynamics are given by

$$
\begin{aligned}
\dot{z}= & \frac{\partial \beta}{\partial \xi} \dot{\xi}+\frac{\partial \beta}{\partial y} x-\bar{C}(y, x) x \\
& +T(y) M(y)^{-1}(F(y, x)+G(y)-\tau)
\end{aligned}
$$

where $\bar{C}(y, x)=\dot{T}(y)-T(y) M(y)^{-1} C(y, x)$.

Assume for now that the Jacobian $\partial \beta / \partial \xi$ is invertiblethis will be confirmed later by the choice of the function $\beta(\cdot)$. Selecting the observer dynamics (3) as in Proposition 1, namely

$$
\begin{aligned}
\dot{\xi}= & -\left(\frac{\partial \beta}{\partial \xi}\right)^{-1}\left(\frac{\partial \beta}{\partial y} \hat{x}-\bar{C}(y, \hat{x}) \hat{x}\right. \\
& \left.+T(y) M(y)^{-1}(F(y, \hat{x})+G(y)-\tau)\right)
\end{aligned}
$$

and using the fact that $\bar{C}(y, x)$ satisfies the linearity property (35) yields the error dynamics

$$
\begin{aligned}
\dot{z}= & -\frac{\partial \beta}{\partial y} L(y) z+\bar{C}(y, L(y) z) \hat{x}+\bar{C}(y, x) L(y) z \\
& -T(y) M(y)^{-1}(F(y, x+L(y) z)-F(y, x))
\end{aligned}
$$

where $L(y)=T(y)^{-1}$.

The transformation matrix $T(y)$ can be obtained by the Cholesky factorisation of the inertia matrix $M(y)$ as introduced in [34], namely $M(y)=T(y)^{\top} T(y)$, yielding

$$
T(y)=\left[\begin{array}{cc}
\sqrt{m_{11}\left(y_{2}\right)} & \frac{m_{12}\left(y_{2}\right)}{\sqrt{m_{11}\left(y_{2}\right)}} \\
0 & \frac{r\left(y_{2}\right)}{m_{11}\left(y_{2}\right)}
\end{array}\right]
$$

where $r\left(y_{2}\right)=\sqrt{m_{11}\left(y_{2}\right)\left(m_{22} m_{11}\left(y_{2}\right)-m_{12}\left(y_{2}\right)^{2}\right)}$. Note that, since the inertia matrix is positive definite, $m_{11}\left(y_{2}\right)>0$ and $m_{22} m_{11}\left(y_{2}\right)-m_{12}\left(y_{2}\right)^{2}>0$, hence the matrix $T(y)$ is invertible. The above selection of $T(y)$ is such that the matrix $\bar{C}(y, x) L(y)=\left[\dot{T}(y)-L(y)^{\top} C(y, x)\right] L(y)$ is skewsymmetric, i.e. $\bar{C}(y, x) L(y)+L(y)^{\top} \bar{C}(y, x)^{\top}=0$. In particular, we have

$$
\bar{C}(y, x) L(y)=\frac{m_{11}{ }^{\prime}\left(y_{2}\right)}{2 r\left(y_{2}\right)}\left(m_{11}\left(y_{2}\right) x_{1}+m_{12}\left(y_{2}\right) x_{2}\right) \mathcal{J}
$$

where

$$
\mathcal{J}=\left[\begin{array}{cc}
0 & -1 \\
1 & 0
\end{array}\right]
$$

Substituting $\hat{x}=L(y) \beta(y, \xi)$ and rearranging the second term on the right-hand side of (38) yields the error dynamics

$$
\begin{aligned}
\dot{z}= & -\Gamma(y, \xi) z+\bar{C}(y, x) L(y) z \\
& -L(y)^{\top}(F(y, x+L(y) z)-F(y, x))
\end{aligned}
$$


where

$$
\begin{gathered}
\Gamma(y, \xi)=\left[\begin{array}{ll}
\gamma_{11}(y, \xi) & \gamma_{12}(y, \xi) \\
\gamma_{21}(y, \xi) & \gamma_{22}(y, \xi)
\end{array}\right] \\
\gamma_{11}(y, \xi)=\frac{1}{\sqrt{m_{11}\left(y_{2}\right)}} \frac{\partial \beta_{1}}{\partial y_{1}}+\frac{m_{11}{ }^{\prime}\left(y_{2}\right) \beta_{2}(y, \xi)}{2 r\left(y_{2}\right)} \\
\gamma_{12}(y, \xi)=\frac{m_{11}\left(y_{2}\right)}{r\left(y_{2}\right)} \frac{\partial \beta_{1}}{\partial y_{2}}-\frac{m_{12}\left(y_{2}\right)}{r\left(y_{2}\right)} \frac{\partial \beta_{1}}{\partial y_{1}} \\
\gamma_{21}(y, \xi)=\frac{1}{\sqrt{m_{11}\left(y_{2}\right)}} \frac{\partial \beta_{2}}{\partial y_{1}}-\frac{m_{11}{ }^{\prime}\left(y_{2}\right) \beta_{1}(y, \xi)}{2 r\left(y_{2}\right)} \\
\gamma_{22}(y, \xi)=\frac{m_{11}\left(y_{2}\right)}{r\left(y_{2}\right)} \frac{\partial \beta_{2}}{\partial y_{2}}-\frac{m_{12}\left(y_{2}\right)}{r\left(y_{2}\right)} \frac{\partial \beta_{2}}{\partial y_{1}} .
\end{gathered}
$$

We now shape the function $\beta(y, \xi)=\left[\beta_{1}(y, \xi), \beta_{2}(y, \xi)\right]^{\top}$ so that the system (39) has a uniformly globally asymptotically stable equilibrium at the origin. To this aim, we first invoke the following (standard) assumption. ${ }^{5}$

Assumption 1: There exists a constant $\nu \in \mathbb{R}$ such that the dissipative force vector $F(y, x)$ satisfies the property

$$
z^{\top} L(y)^{\top} \frac{\partial F(y, x)}{\partial x} L(y) z \geq \nu z^{\top} z
$$

for all $y, x$ and $z$.

Consider now the quadratic Lyapunov function $V(z)=$ $\frac{1}{2} z^{\top} z$ whose time-derivative along the trajectories of (39) satisfies

$$
\begin{aligned}
\dot{V}= & -\gamma_{11}(y, \xi) z_{1}^{2}-\left(\gamma_{12}(y, \xi)+\gamma_{21}(y, \xi)\right) z_{1} z_{2} \\
& -\gamma_{22}(y, \xi) z_{2}^{2}-z^{\top} L(y)^{\top}(F(y, x+L(y) z)-F(y, x)) \\
\leq & -\gamma_{11}(y, \xi) z_{1}^{2}-\left(\gamma_{12}(y, \xi)+\gamma_{21}(y, \xi)\right) z_{1} z_{2} \\
& -\gamma_{22}(y, \xi) z_{2}^{2}-\nu z^{\top} z
\end{aligned}
$$

where we have used (40) combined with the Mean Value Theorem of calculus

$$
\bar{F}(y, x+z)-\bar{F}(y, x)=\left.\frac{\partial \bar{F}(y, x)}{\partial x}\right|_{x=x^{*}} z
$$

where $x^{*}$ is a convex combination of $x$ and $z$. It remains to find functions $\beta_{1}(\cdot)$ and $\beta_{2}(\cdot)$ that solve the PDEs $\gamma_{11}(y, \xi)+$ $\nu>0, \gamma_{12}(y, \xi)+\gamma_{21}(y, \xi)=0$ and $\gamma_{22}(y, \xi)+\nu>0$, and guarantee invertibility of the matrix $\partial \beta / \partial \xi$.

We consider two different cases: 1) when the inertia matrix $M(y)$ has the general form (33) and its entries are bounded functions; and 2) when $M(y)$ is diagonal, i.e. $m_{12}\left(y_{2}\right)=0$, and its entries are not necessarily bounded.

1) Non-diagonal inertia matrix with bounded entries: In this case we assume that the inertia matrix verifies the standard property

$$
M_{\min } I_{2 \times 2} \leq M(y) \leq M_{\max } I_{2 \times 2}
$$

for all $y$ and for some constants $M_{\max } \geq M_{\min }>0$. Selecting $\beta_{1}(\cdot)$ and $\beta_{2}(\cdot)$ as

$$
\begin{aligned}
\beta_{1}(y, \xi)= & \sqrt{m_{11}\left(y_{2}\right)}\left[k_{1}\left(y_{1}+\int_{y_{20}}^{y_{2}} \frac{m_{12}(\zeta)}{m_{11}(\zeta)} \mathrm{d} \zeta\right)\right. \\
& \left.\times\left(k_{2} \pi+\xi_{2}^{2}\right)+\xi_{1}\right] \\
\beta_{2}(y, \xi)= & k_{2} \arctan \left(y_{2}\right)+\xi_{2}
\end{aligned}
$$

${ }^{5}$ Note that Assumption 1 is satisfied for $F(y, x)=\tilde{F}(y) x$, if $\tilde{F}(y)$ is positive definite. leads to $\gamma_{12}(y, \xi)+\gamma_{21}(y, \xi)=0$ and

$$
\begin{aligned}
& \gamma_{11}(y, \xi) \geq-c_{2}\left(k_{2} \arctan \left(y_{2}\right)+\xi_{2}\right)+k_{1}\left(\xi_{2}^{2}+k_{2} \pi\right) \\
& \gamma_{22}(y, \xi) \geq \frac{k_{2} c_{1}}{1+y_{2}^{2}}
\end{aligned}
$$

where the constants $c_{1}$ and $c_{2}$ are such that

$$
c_{1} \leq m_{11}\left(y_{2}\right) / r\left(y_{2}\right) \quad \text { and } \quad c_{2} \geq\left|m_{11}{ }^{\prime}\left(y_{2}\right) /\left(2 r\left(y_{2}\right)\right)\right| .
$$

Note that the existence of such constants is guaranteed by the property (42). The positivity of $\gamma_{11}(\cdot)$ and $\gamma_{22}(\cdot)$ is ensured by picking the "observer gains" $k_{1}$ and $k_{2}$ according to ${ }^{6}$

$$
k_{1} \geq c_{2} \quad k_{2}>\frac{1}{2 \pi} \text {. }
$$

Finally, the Jacobian $\partial \beta / \partial \xi$ is given by

$$
\frac{\partial \beta}{\partial \xi}=\left[\begin{array}{cc}
\sqrt{m_{11}\left(y_{2}\right)} & \star \\
0 & 1
\end{array}\right]
$$

with $\star=2 k_{1} \xi_{2} \sqrt{m_{11}\left(y_{2}\right)}\left(y_{1}+\int_{y_{20}}^{y_{2}} \frac{m_{12}(\zeta)}{m_{11}(\zeta)} \mathrm{d} \zeta\right)$ and it is invertible for any $y_{2}$.

The result is summarised in the following proposition.

Proposition 3: Consider the system (39), where $\beta_{1}(\cdot)$ and $\beta_{2}(\cdot)$ are given by (43) and (44) respectively, and assume that property (42) holds. Then the zero equilibrium of the system (39) is:

(i) uniformly globally stable, if (40) holds with $\nu \geq 0$;

(ii) uniformly globally asymptotically stable, if (40) holds with $\nu \geq 0$ and $\frac{y_{2}(t)}{\epsilon+\sqrt{ } t}$ is bounded for all $t>0$ and for some $\epsilon>0$;

(iii) uniformly globally exponentially stable, if (40) holds with $\nu>0$.

Proof: We will only prove (ii), since (i) and (iii) follow directly from (41). Set $\nu=0$ in (41) and note that as $y_{2}(t)$ tends to infinity, the function $\gamma_{22}(y, \xi)$ tends to zero. Since from $(41)$

$$
\begin{aligned}
|z(t)| \leq & \sqrt{2 V\left(t_{0}\right)} \\
& \times e^{-\frac{1}{2} \int_{t_{0}}^{t} \min \left\{\gamma_{11}(y(\tau), \xi(\tau)), \gamma_{22}(y(\tau), \xi(\tau))\right\} \mathrm{d} \tau}
\end{aligned}
$$

the convergence of $|z(t)|$ to zero is guaranteed if

$$
\lim _{t \rightarrow \infty} \int_{t_{0}}^{t} \gamma_{22}(y(\tau), \xi(\tau)) \mathrm{d} \tau=\lim _{t \rightarrow \infty} \int_{t_{0}}^{t} \frac{k_{2} c_{1}}{1+y_{2}(\tau)^{2}} \mathrm{~d} \tau=\infty .
$$

The above condition is satisfied when $\lim _{t \rightarrow \infty}\left|y_{2}(t)\right| / \sqrt{t} \leq d$, for some finite $d$, namely when $y_{2}(t)$ tends to infinity no faster than $\sqrt{t}$ yielding

$$
|z(t)| \leq \sqrt{2 V\left(t_{0}\right) /(1+t)^{k_{2} c_{1}}} .
$$

If this condition is not verified, then

$$
|z(t)| \leq \sqrt{2 V\left(t_{0}\right) \exp (-\sigma(t))}
$$

where $\sigma(t)=\lim _{t \rightarrow \infty} \int_{t_{0}}^{t} \gamma_{22}(y(\tau), \xi(\tau)) \mathrm{d} \tau<\infty$.

${ }^{6} \mathrm{~A}$ practical guideline is to select the gain $k_{2}$ as $k_{2}=\bar{k}_{2}\left(1+y_{20}^{2}\right)$ with $\bar{k}_{2}>1 /(2 \pi)$, so that large initial values for $y_{2}$ do not affect the initial value of $\gamma_{22}(y, \xi)$. 
2) Diagonal inertia matrix: This is a special case that is encountered, for instance, in the "ball and beam" system as well as in Cartesian manipulators. In this case, we relax condition (42) and replace it with the following assumption.

Assumption 2: There exists a $\mathcal{C}^{r}$ function $f_{2}(\cdot): \mathbb{R} \rightarrow \mathbb{R}$, $r \geq 1$, such that

$$
f_{2}{ }^{\prime}\left(y_{2}\right)>\epsilon \sqrt{m_{22}} \quad \text { and } \quad m_{11}{ }^{\prime}\left(y_{2}\right) f_{2}\left(y_{2}\right) \geq 0
$$

where $\epsilon$ is a positive constant such that $\nu \geq-\epsilon$.

Selecting the functions $\beta_{1}(y, \xi)$ and $\beta_{2}(y, \xi)$ as

$$
\begin{aligned}
& \beta_{1}(y, \xi)=\sqrt{m_{11}\left(y_{2}\right)}\left(k_{1} y_{1}\left(1+\xi_{2}^{2}\right)+\xi_{1}\right) \\
& \beta_{2}(y, \xi)=f_{2}\left(y_{2}\right)+\xi_{2}
\end{aligned}
$$

guarantees that $\gamma_{12}(y, \xi)+\gamma_{21}(y, \xi)=0$ and

$$
\begin{aligned}
& \gamma_{11}(y, \xi) \geq-c_{2} \xi_{2}+k_{1}\left(1+\xi_{2}^{2}\right) \\
& \gamma_{22}(y, \xi)=\frac{1}{\sqrt{m_{22}}} f_{2}{ }^{\prime}\left(y_{2}\right)>\epsilon>0
\end{aligned}
$$

where $c_{2} \geq\left|m_{11}{ }^{\prime}\left(y_{2}\right) /\left(2 r\left(y_{2}\right)\right)\right|>0$ and we have used Assumption 2. Choosing the "gain" $k_{1}$ according to

$$
k_{1}>\frac{\epsilon+\sqrt{\epsilon^{2}+c_{2}^{2}}}{2}
$$

ensures that $\gamma_{11}(y, \xi)>\epsilon$ for all $y$ and $\xi$, hence the derivative of the Lyapunov function given in (41) is negative definite. Finally, note that the Jacobian matrix $\partial \beta / \partial \xi$ is invertible, in particular

$$
\frac{\partial \beta}{\partial \xi}=\left[\begin{array}{cc}
\sqrt{m_{11}\left(y_{2}\right)} & 2 k_{2} y_{1} \xi_{2} \sqrt{m_{11}\left(y_{2}\right)} \\
0 & 1
\end{array}\right]
$$

The result is summarised in the following proposition.

Proposition 4: Consider the system (39), where $\beta_{1}(\cdot)$ and $\beta_{2}(\cdot)$ are given by (45) and (46) respectively, and suppose that Assumptions 1 and 2 hold. Then the system (39) has a uniformly globally exponentially stable equilibrium at the origin.

\section{Simulation results}

In this section the observers developed in Sections IV-B1 and IV-B2 are tested via simulations respectively on a two-link manipulator with revolute joints and on the "ball and beam" system.

1) Two-link manipulator: We consider a revolute-joints arm with two links and with a mass attached to the extremum of each link [30]. The inertia matrix $M(y)$ and the vector $G(y)$ of the two-link manipulator are given by

$$
\begin{aligned}
M(y) & =\left[\begin{array}{cc}
a_{1}+a_{2} \cos \left(y_{2}\right) & \frac{1}{2} a_{2} \cos \left(y_{2}\right)+a_{3} \\
\frac{1}{2} a_{2} \cos \left(y_{2}\right)+a_{3} & a_{3}
\end{array}\right] \\
G(y) & =\left[\begin{array}{c}
g_{1} \cos \left(y_{1}\right)+g_{2} \cos \left(y_{1}+y_{2}\right) \\
g_{2} \cos \left(y_{1}+y_{2}\right)
\end{array}\right]
\end{aligned}
$$

where $y_{i}$ is the angle measured at the $i$-th revolute joint. The model parameters are given by

$$
\begin{aligned}
a_{1} & =I_{1}+I_{2}+m_{1} L_{c 1}^{2}+m_{2}\left(L_{1}^{2}+L_{c 2}^{2}\right) \\
a_{2} & =2 m_{2} L_{c 2} L_{1} \\
a_{3} & =m_{2} L_{c 2}^{2}+I_{2} \\
g_{1} & =\left(L_{c 1}\left(m_{1}+M_{1}\right)+L_{1}\left(m_{2}+M_{2}\right)\right) g \\
g_{2} & =L_{c 2}\left(m_{2}+M_{2}\right) g \\
L_{c 1} & =L_{1}\left(m_{1} / 2+M_{1}\right) /\left(m_{1}+M_{1}\right) \\
L_{c 2} & =L_{2}\left(m_{2} / 2+M_{2}\right) /\left(m_{2}+M_{2}\right) \\
I_{1} & =\left(L_{1}^{2}+L_{w 1}^{2}\right)\left(m_{1}+M_{1}\right) / 12 \\
I_{2} & =\left(L_{2}^{2}+L_{w 2}^{2}\right)\left(m_{2}+M_{2}\right) / 12
\end{aligned}
$$

where $g$ is the gravitational acceleration, $M_{i}$ is the mass attached to link $i$ and $m_{i}, L_{i}, L_{w i}, L_{c i}$, and $I_{i}$ denote respectively the mass, length, width, centre of mass, and moment of inertia for each link $i$. The matrix for the Coriolis and centripetal forces evaluated through the Christoffel symbols is

$$
C(y, x)=\left[\begin{array}{cc}
-\frac{1}{2} a_{2} \sin \left(y_{2}\right) x_{2} & -\frac{1}{2} a_{2} \sin \left(y_{2}\right)\left(x_{1}+x_{2}\right) \\
\frac{1}{2} a_{2} \sin \left(y_{2}\right) x_{1} & 0
\end{array}\right] .
$$

The transformation matrix $T(y)$ is given by

$$
T(y)=\left[\begin{array}{cc}
\sqrt{a_{1}+a_{2} \cos \left(y_{2}\right)} & \frac{a_{2} \cos \left(y_{2}\right)+2 a_{3}}{2 \sqrt{a_{1}+a_{2} \cos \left(y_{2}\right)}} \\
0 & \sqrt{\frac{4 a_{3} a_{1}-a_{2}^{2}\left(\cos \left(y_{2}\right)\right)^{2}-4 a_{3}^{2}}{4 a_{1}+4 a_{2} \cos \left(y_{2}\right)}}
\end{array}\right] .
$$

The functions $\beta_{1}(\cdot)$ and $\beta_{2}(\cdot)$, evaluated directly through (43) and (44), are

$$
\begin{aligned}
\beta_{1}(y, \xi)= & \sqrt{a_{1}+a_{2} \cos \left(y_{2}\right)}\left[k_{1}\left(k_{2} \pi+\xi_{2}^{2}\right)\right. \\
& \left.\times\left(y_{1}+\int_{y_{20}}^{y_{2}} \frac{a_{2} \cos (\zeta)+2 a_{3}}{2 a_{1}+2 a_{2} \cos (\zeta)} \mathrm{d} \zeta\right)+\xi_{1}\right] \\
\beta_{2}(y, \xi)= & k_{2} \arctan \left(y_{2}\right)+\xi_{2} .
\end{aligned}
$$

Assuming that there is no a priori knowledge about initial velocities of the links, the initial conditions of the observer states $\left(\xi_{10}, \xi_{20}\right)$ are chosen so that the initial estimate $\hat{x}_{0}$ is zero, i.e.

$$
\begin{aligned}
& \xi_{20}=-k_{2} \arctan \left(y_{20}\right) \\
& \xi_{10}=-k_{1}\left(k_{2} \pi+\xi_{20}^{2}\right) y_{10} .
\end{aligned}
$$

Only the case of zero friction is considered, i.e. $\nu=0$. The model parameters are set to $L_{1}=0.6 \mathrm{~m}, m_{1}=1 \mathrm{~kg}, M_{1}=$ $1 \mathrm{~kg}, L_{w 1}=0.07 \mathrm{~m}, L_{2}=0.4 \mathrm{~m}, m_{2}=0.6 \mathrm{~kg}, M_{2}=0.8 \mathrm{~kg}$, $L_{w 2}=0.07 \mathrm{~m}$, and $g=9.81 \mathrm{~m} / \mathrm{s}^{2}$, yielding $a_{1}=0.558$, $a_{2}=0.288, a_{3}=0.078, g_{1}=17.02$ and $g_{2}=4.32$.

Two simulation results are shown for different observer gains. The initial states of the two-link manipulator are $X=\left(y_{10}, y_{20}, x_{10}, x_{20}\right)=(0,0,1.5,1.5)$, with motor torque $\tau=0$. The state trajectories of the two-link arm with the given initial conditions are depicted in Figure 1. Figure 2 shows the estimation errors for different values of $k_{1}$ and $k_{2}$. It is evident, as suggested by the Lyapunov function derivative, that the observer gain $k_{1}$ mainly affects the convergence to zero of the error $z_{1}$, whereas $k_{2}$ affects the convergence of both $z_{1}$ and $z_{2}$. 


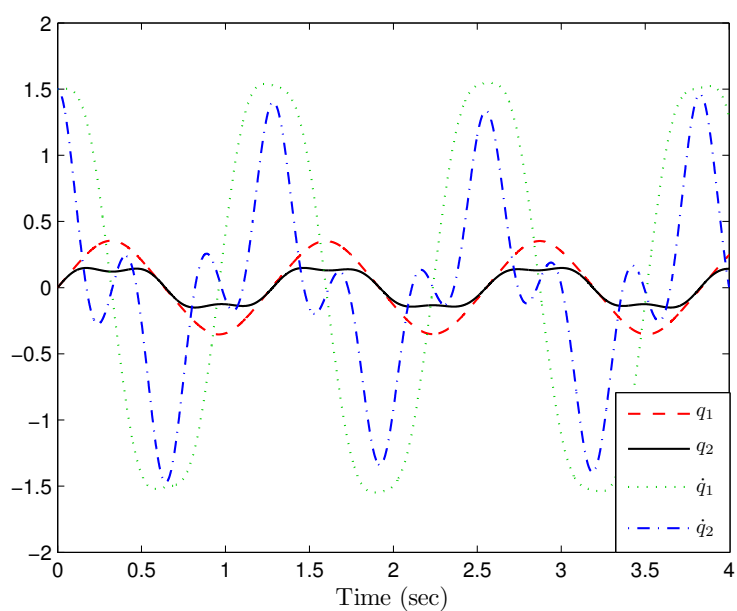

Fig. 1. Unforced trajectories of the two-link manipulator.
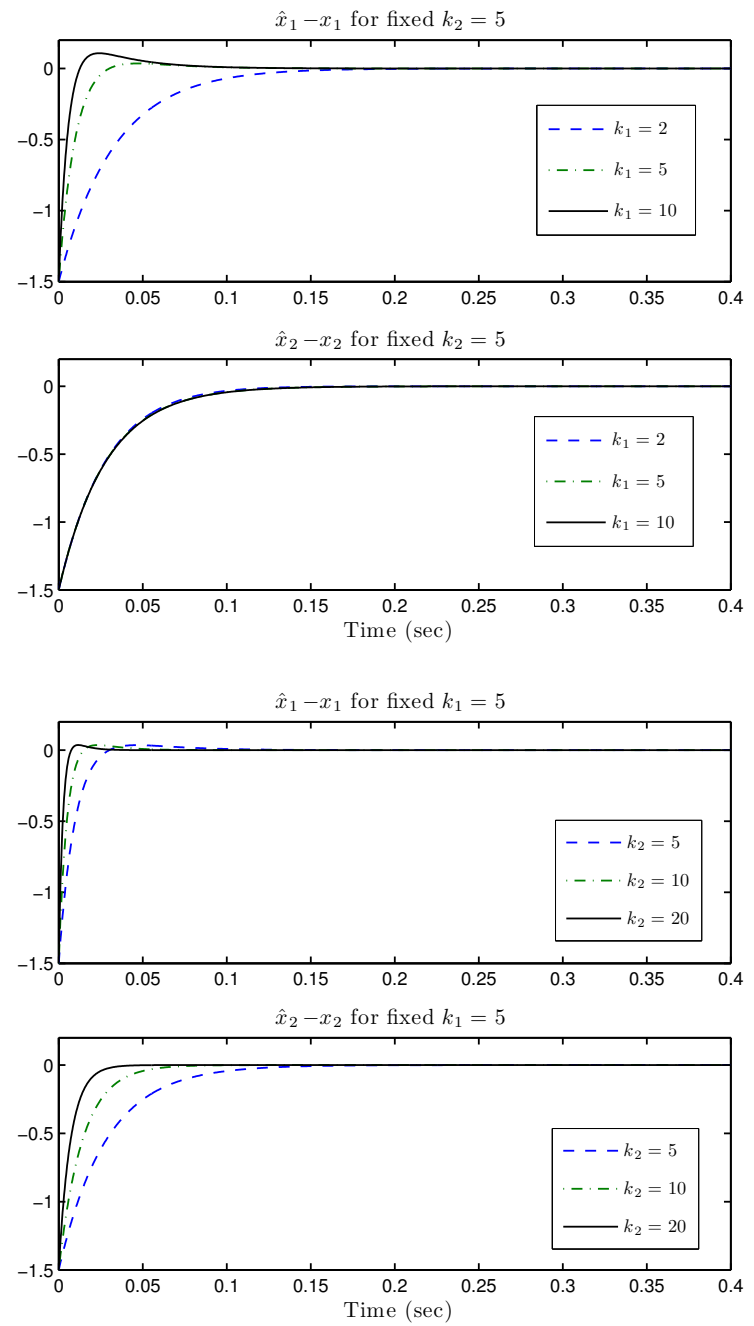

Fig. 2. Time histories of the observer errors $\hat{x}_{1}-x_{1}$ and $\hat{x}_{2}-x_{2}$ for the two-link manipulator.
2) Ball and beam system: We consider the ball and beam system [31], [32] which is described by the equations (36)(37), where the states $y_{1}$ and $y_{2}$ correspond to the position of the ball and the angle of the beam respectively, $F(y, x)=0$, and

$$
\begin{aligned}
M(y) & =\left[\begin{array}{cc}
B+m y_{2}^{2} & 0 \\
0 & A
\end{array}\right] \quad C(y, x)=\left[\begin{array}{cc}
0 & 2 m y_{2} x_{1} \\
-m y_{2} x_{1} & 0
\end{array}\right] \\
G(y) & =\left[\begin{array}{c}
m g y_{2} \cos \left(y_{1}\right) \\
m g \sin \left(y_{1}\right)
\end{array}\right]
\end{aligned}
$$

where $A=\frac{J_{b}}{R^{2}}+m, B=J+J_{b}, m, R$ and $J_{b}$ are the mass, radius and moment of inertia of the ball, respectively, $J$ is the moment of inertia of the beam, $g$ is the acceleration due to gravity, and $\tau$ is the applied torque.

The functions $\beta_{1}(y, \xi)$ and $\beta_{2}(y, \xi)$ are obtained from (45) and (46) as

$$
\begin{aligned}
& \beta_{1}(y, \xi)=\sqrt{B+m y_{2}^{2}}\left(k_{1} y_{1}\left(1+\xi_{2}^{2}\right)+\xi_{1}\right) \\
& \beta_{2}(y, \xi)=k_{2}\left(\frac{1}{3} y_{2}^{3}+y_{2}\right)+\xi_{2}
\end{aligned}
$$

where we have selected the function $f_{2}\left(y_{2}\right)$ to satisfy Assumption 2. Note that the derivative of the Lyapunov function $V(z)=\frac{1}{2} z^{\top} z$ satisfies

$$
\dot{V}(z) \leq-\left(k_{1}\left(1+\xi_{2}^{2}\right)-c_{2}\left|\xi_{2}\right|\right) z_{1}^{2}-k_{2}\left(1+y_{2}^{2}\right) z_{2}^{2}
$$

and is rendered negative definite by selecting $k_{1} \geq c_{2}$.

Simulations of the unforced $(\tau=0)$ ball and beam system and the observer described above have been carried out for two different sets of initial conditions, namely $X_{1}=(0.05,0,0,0)$ and $X_{2}=(-0.2,0,0.7227426878192512,0)$. We have used the same parameter values as in [32], namely $m=0.05$ $\mathrm{kg}, R=0.01 \mathrm{~m}, J_{b}=2 \cdot 10^{-6} \mathrm{~kg} \mathrm{~m}{ }^{2}, J=0.02 \mathrm{~kg}$ $\mathrm{m}^{2}$, and $g=9.81 \mathrm{~m} / \mathrm{s}^{2}$. The response of the system states to the initial conditions $X_{1}$ and $X_{2}$ are shown in Figure 3. Notice that for the initial condition $X_{1}$ the position of the ball diverges, while $X_{2}$ has been chosen so that the system exhibits an initial oscillatory behaviour. The time histories of the observer error variables $\hat{x}_{1}-x_{1}$ and $\hat{x}_{2}-x_{2}$, for different values of the parameters $k_{1}$ and $k_{2}$, are shown in Figures $4-5$. We see that the errors converge asymptotically to zero at a rate that increases with $k_{1}$ and $k_{2}$. From (47) it is clear that incrementing $k_{1}$ leads to a higher decaying rate of both observer errors, whereas incrementing $k_{2}$ leads to an increment of the decaying rate for the observer error $z_{2}$ which corresponds to the angular velocity of the beam.

\section{Conclusions}

The problem of constructing globally convergent (reducedorder) observers for nonlinear systems has been addressed. A general framework has been developed, which relies on rendering attractive an appropriately selected invariant manifold in the extended space of the plant and the observer. The benefit of introducing this manifold is that it leads to a novel definition of the "state estimation error" and provides extra "controls" with which to stabilise the observer error dynamics.

It has been shown that the proposed approach can be applied to systems with non-monotonic nonlinearities, thus 

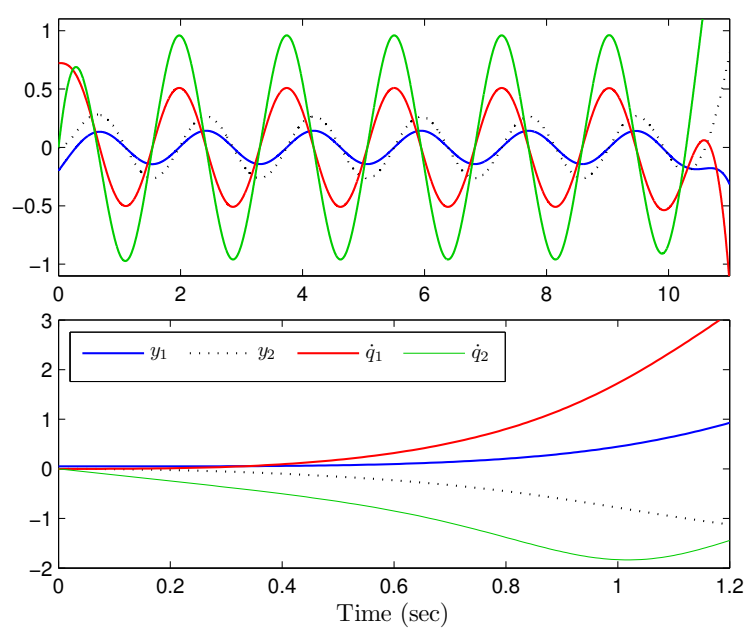

Fig. 3. Unforced response of the ball and beam system for the initial conditions $X_{1}$ (bottom) and $X_{2}$ (top).
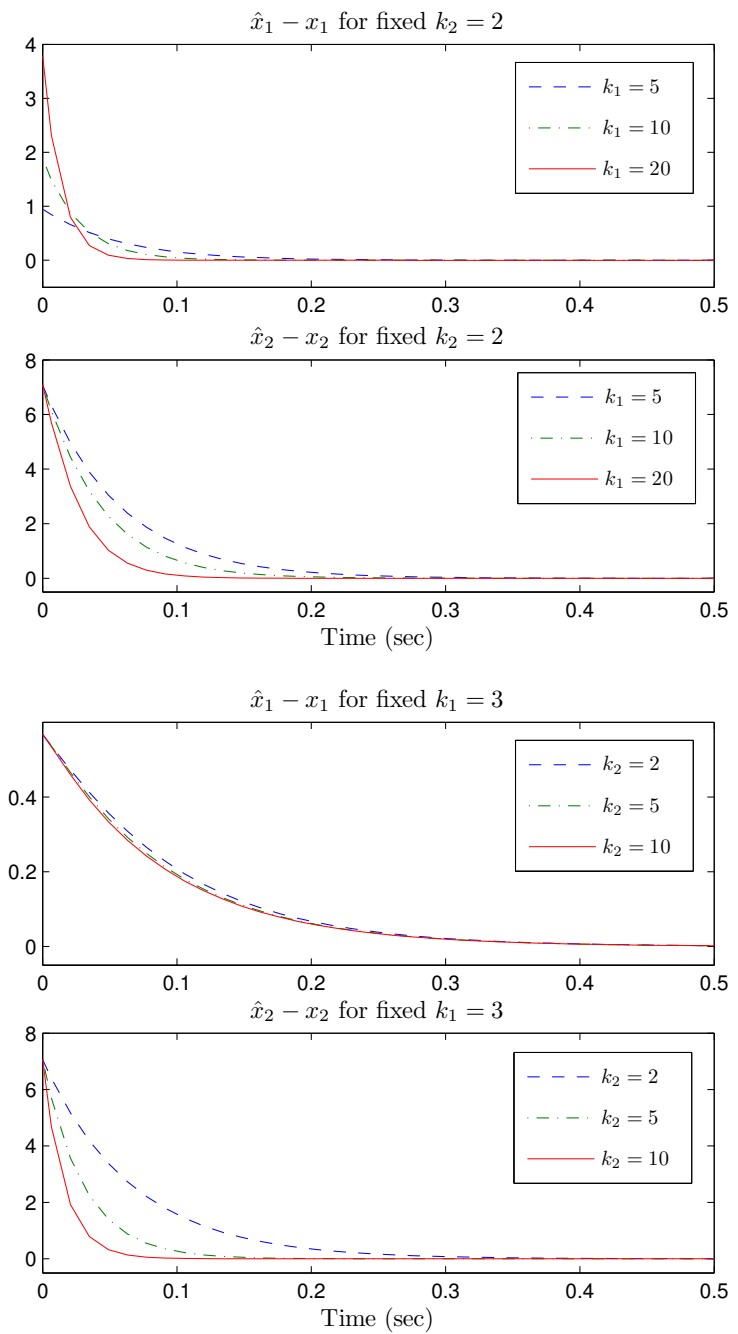

Fig. 4. Time histories of the observer errors $\hat{x}_{1}-x_{1}$ and $\hat{x}_{2}-x_{2}$ for the ball and beam system and for initial conditions $X_{1}$.
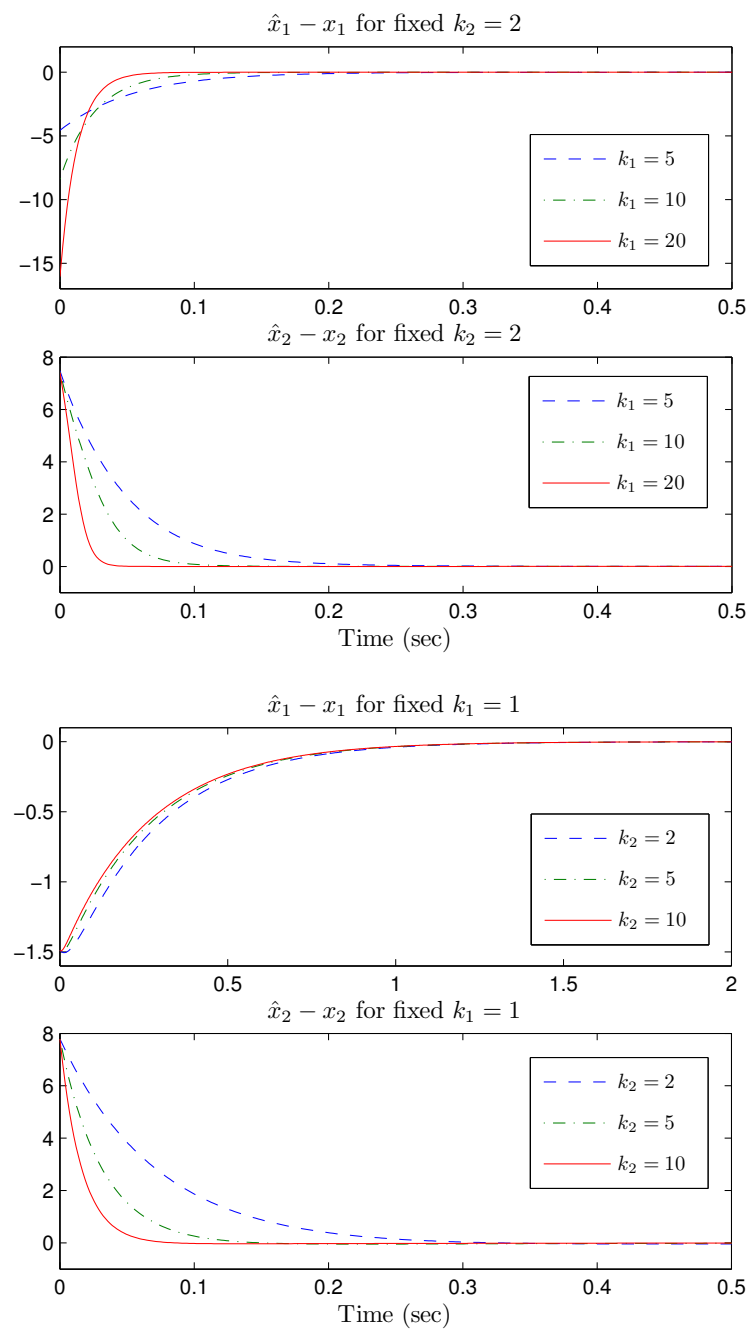

Fig. 5. Time histories of the observer errors $\hat{x}_{1}-x_{1}$ and $\hat{x}_{2}-x_{2}$ for the ball and beam system and for initial conditions $X_{2}$.

significantly enlarging the class of systems considered in [17] and related works. Moreover, in the case of systems with monotonic nonlinearities it generalises the result in [17].

The method has been used to obtain new solutions to the problems of range identification for perspective vision systems and velocity estimation for 2DOF Euler-Lagrange systems.

Further research is underway to exploit the results in the design of functional observers, in the design of observers for hybrid systems, and to assess the sensitivity of the observer in the presence of measurement noise and unmodelled dynamics.

\section{REFERENCES}

[1] D. Luenberger, "An introduction to observers," IEEE Trans. Automatic Control, vol. 16, no. 6, pp. 596-602, 1971.

[2] R. Engel and G. Kreisselmeier, "A continuous-time observer which converges in finite time," IEEE Trans. Automatic Control, vol. 47, no. 7, pp. 1202-1204, 2002.

[3] A. Krener and A. Isidori, "Linearization by output injection and nonlinear observers," Systems \& Control Letters, vol. 3, no. 1, pp. 47-52, 1983.

[4] A. Krener and W. Respondek, "Nonlinear observers with linearizable error dynamics," SIAM J. Control and Optimization, vol. 23, no. 2, pp. 197-216, 1985.

[5] A. Krener and M. Xiao, "Observers for linearly unobservable nonlinear systems," Systems \& Control Letters, vol. 46, no. 4, pp. 281-288, 2002. 
[6] J. Tsinias, "Further results on the observer design problem," Systems \& Control Letters, vol. 14, no. 5, pp. 411-418, 1990.

[7] J. Gauthier, H. Hammouri, and S. Othman, "A simple observer for nonlinear systems applications to bioreactors," IEEE Trans. Automatic Control, vol. 37, no. 6, pp. 875-880, 1992.

[8] J. Gauthier and I. Kupka, "Observability and observers for nonlinear systems," SIAM J. Control and Optimization, vol. 32, no. 4, pp. 975994, 1994.

[9] G. Ciccarella, M. Dalla Mora, and A. Germani, "A Luenberger-like observer for nonlinear systems," Int. J. Control, vol. 57, no. 3, pp. 537556, 1993.

[10] N. Kazantzis and C. Kravaris, "Nonlinear observer design using Lyapunov's auxiliary theorem," Systems \& Control Letters, vol. 34, no. 5, pp. 241-247, 1998

[11] A. Krener and M. Xiao, "Nonlinear observer design in the Siegel domain," SIAM J. Control and Optimization, vol. 41, no. 3, pp. 932-953, 2002.

[12] V. Andrieu and L. Praly, "On the existence of a Kazantzis-Kravaris/ Luenberger observer," SIAM J. Control and Optimization, vol. 45, no. 2, pp. 432-456, 2006.

[13] G. Kreisselmeier and R. Engel, "Nonlinear observers for autonomous Lipschitz continuous systems," IEEE Trans. Automatic Control, vol. 48, no. 3, pp. 451-464, 2003.

[14] A. Astolfi and L. Praly, "Global complete observability and output-tostate stability imply the existence of a globally convergent observer," Math. Control, Signals, and Systems, vol. 18, no. 1, pp. 32-65, 2006.

[15] H. Lei, J. Wei, and W. Lin, "A global observer for observable autonomous systems with bounded solution trajectories," in Proc. 44th IEEE Conf. Decision and Control and European Control Conference, Seville, Spain, 2005, pp. 1911-1916.

[16] H. Khalil, "High-gain observers in nonlinear feedback control," in New Directions in Nonlinear Observer Design, H. Nijmeijer and T. Fossen, Eds. Springer-Verlag, Berlin, 1999.

[17] M. Arcak and P. Kokotović, "Nonlinear observers: a circle criterion design and robustness analysis," Automatica, vol. 37, no. 12, pp. 19231930, 2001.

[18] — , "Observer-based control of systems with slope-restricted nonlinearities," IEEE Trans. Automatic Control, vol. 46, no. 7, pp. 1146-1150, 2001.

[19] X. Fan and M. Arcak, "Observer design for systems with multivariable monotone nonlinearities," Systems \& Control Letters, vol. 50, no. 4, pp. 319-330, 2003

[20] D. Karagiannis and A. Astolfi, "Nonlinear observer design using invariant manifolds and applications," in Proc. 44th IEEE Conf. Decision and Control and European Control Conference, Seville, Spain, 2005, pp. $7775-7780$.

[21] A. Astolfi and R. Ortega, "Immersion and invariance: a new tool for stabilization and adaptive control of nonlinear systems," IEEE Trans. Automatic Control, vol. 48, no. 4, pp. 590-606, 2003.

[22] D. Karagiannis, A. Astolfi, and R. Ortega, "Two results for adaptive output feedback stabilization of nonlinear systems," Automatica, vol. 39, no. 5, pp. 857-866, 2003.

[23] D. Carnevale, D. Karagiannis, and A. Astolfi, "Reduced-order observer design for systems with non-monotonic nonlinearities," in Proc. 45th IEEE Conf. Decision and Control, San Diego, California, 2006.

[24] M. Jankovic and B. Ghosh, "Visually guided ranging from observations of points, lines and curves via an identifier based nonlinear observer," Systems \& Control Letters, vol. 25, no. 1, pp. 63-73, 1995.

[25] X. Chen and H. Kano, "A new state observer for perspective systems," IEEE Trans. Automatic Control, vol. 47, no. 4, pp. 658-663, 2002.

[26] W. Dixon, Y. Fang, D. Dawson, and T. Flynn, "Range identification for perspective vision systems," IEEE Trans. Automatic Control, vol. 48, no. 12 , pp. 2232-2238, 2003.

[27] D. Karagiannis and A. Astolfi, "A new solution to the problem of range identification in perspective vision systems," IEEE Trans. Automatic Control, vol. 50, no. 12, pp. 2074-2077, 2005.

[28] N. Aghannan and P. Rouchon, "An intrinsic observer for a class of Lagrangian systems," IEEE Trans. Automatic Control, vol. 48, no. 6, pp. $936-945,2003$.

[29] G. Besançon, "Global output feedback tracking control for a class of Lagrangian systems," Automatica, vol. 36, no. 12, pp. 1915-1921, 2000.

[30] M. Spong, S. Hutchinson, and M. Vidyasagar, Robot Modeling and Control. John Wiley and Sons, 2006.

[31] J. Hauser, S. Sastry, and P. Kokotović, "Nonlinear control via approximate input-output linearization: the ball and beam example," IEEE Trans. Automatic Control, vol. 37, no. 3, pp. 392-398, 1992.
[32] J. Huang and C. Lin, "Robust nonlinear control of the ball and beam system," in Proc. American Control Conference, Seattle, Washington, 1995, pp. 306-310.

[33] B. Siciliano and L. Villani, Robot Force Control. Kluwer Academic Publishers, 2000.

[34] A. Jain and G. Rodriguez, "Diagonalized Lagrangian robot dynamics," IEEE Trans. Robotics and Automation, vol. 11, no. 4, pp. 571-584, 1995.

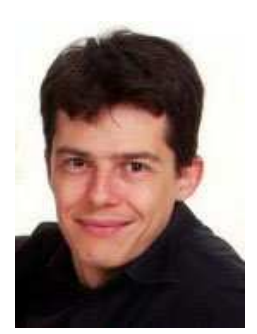

Dimitrios Karagiannis (M'06) was born in 1976. He received his degree in Electrical and Computer Engineering from the Aristotle University of Thessaloniki, Greece, in 2000, the M.Sc. with distinction in Control Systems from Imperial College London, UK, in 2001, and his Ph.D. degree from the University of London in 2005. He has been a visiting research student at the Laboratoire des Signaux et Systèmes (SUPELEC) in Paris, France, and at the Laboratoire de Génie Electrique de Paris. He is coauthor of 9 journal papers, 2 book chapters and 15 papers in peer-reviewed conference proceedings. He is also co-author of the book "Nonlinear and Adaptive Control with Applications", Springer-Verlag, 2008. He is currently a Research Associate at Imperial College London. His area of research is nonlinear and adaptive control design with applications to electrical, mechanical and electromechanical systems and in particular power converters, electric machines and flight control systems.

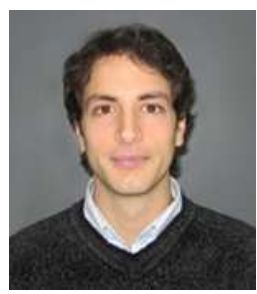

Daniele Carnevale was born in Italy in $1978 . \mathrm{He}$ graduated with a degree in electrical engineering from the University of Rome "Tor Vergata" in 2003. $\mathrm{He}$ received his Ph.D. degree in Robotics applied to surgery at the same university in 2007, where he is currently a research assistant. He was a visiting scholar at the Center for Control Engineering and Computation, University of California, Santa Barbara, in 2005, and at the Electrical and Electronic Engineering Department, Imperial College, London (UK), in 2006. His research interests are focused on nonlinear observer design, mathematical modeling of hysteresis, extremum seeking and networked controlled systems.

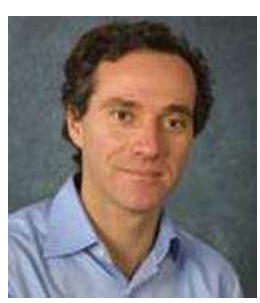

Alessandro Astolfi (M'98-SM'02) was born in Rome, Italy, in 1967. He graduated in electrical engineering from the University of Rome in 1991. In 1992 he joined ETH-Zurich where he obtained a M.Sc. in Information Theory in 1995 and the Ph.D. degree with Medal of Honour in 1995 with a thesis on discontinuous stabilization of nonholonomic systems. In 1996 he was awarded a Ph.D. from the University of Rome "La Sapienza" for his work on nonlinear robust control. Since 1996 he is with the Electrical and Electronic Engineering Department of Imperial College, London (UK), where he is currently Professor in Non-linear Control Theory. From 1998 to 2003 he was also an Associate Professor at the Dept. of Electronics and Information of the Politecnico of Milano. Since 2005 he is also Professor at Dipartimento di Informatica, Sistemi e Produzione, University of Rome "Tor Vergata". He has been visiting lecturer in "Nonlinear Control" in several universities, including ETH-Zurich (1995-1996); Terza University of Rome (1996); Rice University, Houston (1999); Kepler University, Linz (2000); SUPELEC, Paris (2001). His research interests are focused on mathematical control theory and control applications, with special emphasis on the problems of discontinuous stabilization, robust stabilization, robust control and adaptive control. In 2007 he was awarded the IEEE Control Systems Society Antonio Ruberti Young Researcher Prize. The Prize is given annually to "recognize distinguished cutting-edge contributions by a young researcher to the theory or application of systems and control". $\mathrm{He}$ is author of more than 70 journal papers, of 20 book chapters and of over 160 papers in refereed conference proceedings. He is author (with D. Karagiannis and R. Ortega) of the monograph "Nonlinear and Adaptive Control with Applications" (Springer-Verlag). He is Associate Editor of Systems and Control Letters, Automatica, IEEE Trans. Automatic Control, the International Journal of Control, the European Journal of Control, the Journal of the Franklin Institute, and the International Journal of Adaptive Control and Signal Processing. He has also served in the IPC of various international conferences. 